\title{
Magnetic anisotropy in ordered textured Co nanowires
}

\author{
L. G. Vivas, ${ }^{1}$ J. Escrig, ${ }^{2}$ D. G. Trabada, ${ }^{1}$ G. A. Badini-Confalonieri, ${ }^{1}$ \\ and M. Vázquez ${ }^{1}$ \\ ${ }^{1}$ Institute of Materials Science of Madrid, CSIC. 28049 Madrid, Spain \\ ${ }^{2}$ Departamento de Física, Universidad de Santiago de Chile (USACH), and Center for the Development of \\ Nanoscience and Nanotechnology (CEDENNA), Avda. Ecuador 3493, 9170124 Santiago, Chile
}

(Received 2 February 2012; accepted 1 June 2012; published online 19 June 2012)

\begin{abstract}
The magnetization reversal in ordered arrays of Co nanowires with tailored $h c p$-phase texture, controlled by $\mathrm{pH}$ synthesis and nanowires length, has been investigated. The angular dependence of coercivity has been experimentally determined for different crystal textures, and the corresponding magnetization reversal mode is interpreted by analytical modelling. The results show that reversal takes place by propagation of a transverse-like domain wall mode. The fitting of experimental and calculated data allows us the quantitative evaluation of the magnetocrystalline anisotropy constant strength whose magnetization easy direction evolves from parallel to the wires toward in-plane orientation with the change of $h c p$-phase texture. (C) 2012 American Institute of Physics. [http://dx.doi.org/10.1063/1.4729782]
\end{abstract}

The simple geometry and high aspect ratios of arrays of magnetic nanowires make them a model system for the study of magnetic phenomena in uniaxial nanomagnets for modern devices applications. ${ }^{1,2}$ While ultrasoft magnetic nanowires (i.e., permalloy) have been exhaustively investigated, Co nanowires form a particularly interesting system as it is a hard magnetic material which magnetic properties strongly depend on their crystal structure (i.e., phases, texture, and grain size). The control of the orientation of $h c p-c$ axis (i.e., magnetization easy axis of magnetocristalline anisotropy, $K_{m c}$ ) results in the control of the Co nanowires effective anisotropy. A strong longitudinal magnetic anisotropy is achieved when the $c$ axis is oriented parallel to the nanowires, so reinforcing the shape anisotropy. Since the magnetization reversal process is determined by the strength and orientation of the effective magnetic anisotropy, its detailed control and understanding will benefit advances in those applications.

In Co nanowires prepared by electrochemical route, crystalline structure can be tuned by adjusting fabrication parameters as current density, plating time, $p \mathrm{H}$, pore diameter, or annealing and deposition under external magnetic fields. ${ }^{3-7}$ Particularly, it has been shown that $p \mathrm{H}$-controlled electroplating enables the switching between $f c c$ and $h c p$-Co phases, which modifies the magnetization easy axis from parallel to perpendicular to the wires. ${ }^{6,7}$ This is typically qualitatively concluded from the differences in the hysteresis loops shape between parallel and perpendicular applied magnetic field configurations.

To achieve full understanding of the magnetization reversal defined by a given anisotropy, the study of coercivity and, specifically of its angular dependence, is an useful tool. Different reversal modes can be induced by suitable modification of the $K_{m c}$ parameter. This feature is relevant for the design of hybrid systems, as multilayers of different Co crystallographic structures, and consequently different controlled reversal modes, which is of interest in spintronic and microwaves devices. Previous works on $\mathrm{Co} / \mathrm{Cu}$ multilayer nanowires ${ }^{8}$ show that competing anisotropies can be present in nanowires. The control of magnetic anisotropy in these nanostructured systems is very important both for potential use of this material in technological applications as well as for the interpretation of experimental results and the basic magnetic understanding. It should be mentioned that the effective magnetic anisotropy is a resultant of the contributions from magnetocrystalline and shape anisotropy terms. Different methods can be used to experimentally estimate the effective anisotropy field, such as ferromagnetic resonance, ${ }^{9}$ ac susceptibility, ${ }^{10}$ or magnetometric techniques. ${ }^{11}$

In this work, we present a combined experimental and analytical calculations investigation of the angular dependent magnetization reversal in Co nanowires with different $K_{m c}$ contributions, which depend on the plating bath $p \mathrm{H}$ and time (i.e., nanowires length).

Nanowires were synthesized via dc electroplating into pore channels of self-standing oxalic acid nanoporous oxide aluminium membranes (AAO) prepared by doubleanodization method. ${ }^{5}$ Electrodeposition was carried out using an aqueous electrolyte containing $250 \mathrm{~g} / \mathrm{C} \mathrm{CoSO}_{4}$ and $40 \mathrm{~g} / \mathrm{l} \mathrm{H}_{3} \mathrm{BO}_{3}$ at room temperature, under constant stirring, and at a bias of $-1 \mathrm{~V}$ (versus $\mathrm{Ag} / \mathrm{AgCl}$ ). The as-prepared solution has typical $p \mathrm{H}$ value of 3.5 , which has been gradually increased up to 6.7 by the addition of diluted $\mathrm{NaOH}$. The wires grew in the usual bottom-up fashion starting from the $\mathrm{Au}$ electrode at the pore bottoms and the average wire length $(L)$ was controlled by monitoring the total charge that passed during deposition. Scanning electron microscopy (SEM) allowed us to determine the pore diameter $(50 \mathrm{~nm})$, the interpore distance $(105 \mathrm{~nm})$, and the length of the wires, tailored to be from $3 \mu \mathrm{m}$ to $30 \mu \mathrm{m}$. SEM images in Fig. 1(a) of the lateral view of the AAO membrane filled with nanowires and of its surface confirm the hexagonal order degree and the homogeneity of the wires.

$\mathrm{X}$-ray diffraction (XRD) patterns of nanowires embedded into the templates and electroplated at three different $p \mathrm{H}$ values: $3.5,5.0$, and 6.7 are presented in Fig. 1(b) for $L \sim 3 \mu \mathrm{m}$. The $h c p$-phase is identified in all the cases, showing strong textures along [100], [101], and [002] directions 


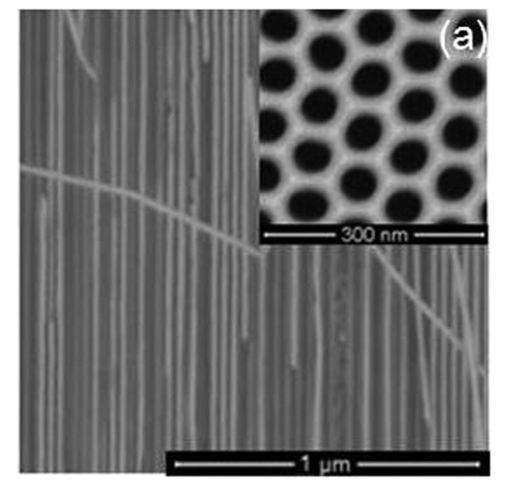

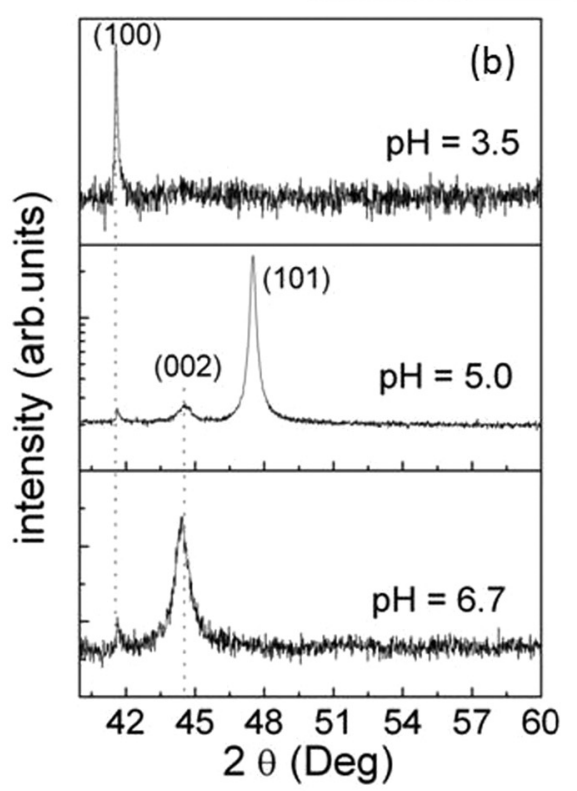

depending on the respective $p \mathrm{H}$ value. These textured structures at $2 \theta=41.5^{\circ}, 47.5^{\circ}$, and $44.5^{\circ}$ are ascribed to hexagonal crystalline anisotropy with $c$-axis nearly perpendicular, at $\sim 45^{\circ}$ and parallel to the wires axis, respectively. ${ }^{5,6}$ However, increasing the length of the wires leads to a reduction of the crystal texture, particularly for the (002) peak as can be observed in Fig. 1(c).

Magnetic hysteresis loops, shown in Fig. 2 for arrays of Co nanowires $3 \mu \mathrm{m}$ long, have been measured in a vibrating sample magnetometer (VSM) under longitudinal (//) (parallel to nanowires axis) and perpendicular $(\perp)$ (in-plane) configuration of the applied field. The saturation magnetization, $\mathbf{M}_{\mathrm{s}}$, values obtained from these measurements differ by $15 \%$ to $20 \%$ of the Co bulk value $\left(1440 \mathrm{emu} / \mathrm{cm}^{3}\right) .{ }^{12}$ This reduction can be ascribed not only to the nanostructuring of the sample but also to some error when estimating the exact volume of magnetic material as well as to the possibility of loss of material due to oxidation. ${ }^{13}$

A clear uniaxial magnetic anisotropy can be inferred from the high longitudinal remanence and coercivity and their vanishing perpendicular values for the nanowires textured in the [002] crystallographic direction. The nanowires textured in the [101] direction show a deviation of the easy axis from the wire axis, while a more complex distribution of anisotropies is suggested for the [100] texture.

In order to analyze in further detail, the actual magnetization reversal processes, the full angular dependence of hysteresis loops was measured. The experimental coercivity $\left(H_{c}\right)$ can be compared with the expected evolution assuming different possible reversal modes: coherent $(\mathrm{C})$, transverse $(T)$, and vortex $(V)$ wall reversal modes. While the coherent mode corresponds to homogeneous magnetization rotation along the entire wire, the transverse wall mode is observed in long and thin enough wires where the exchange interaction forces the magnetization to be homogeneous through a radial cross section of the cylinder. ${ }^{14}$ The vortex reversal mode occurs in wires of larger diameter, when the remanent state is not a pure flower-state but contains also a pronounced vortex component, which develops in order to reduce magnetic surface charges.
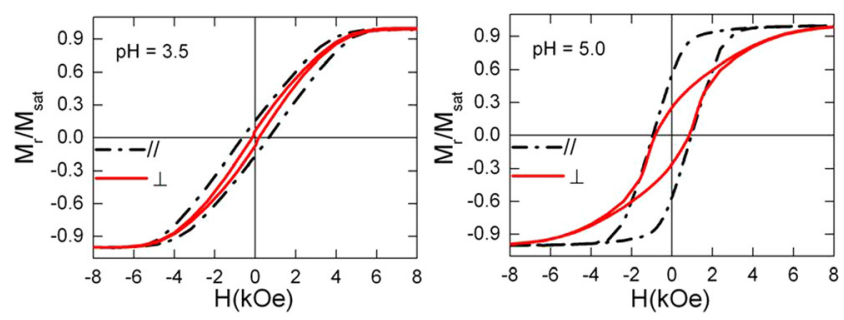

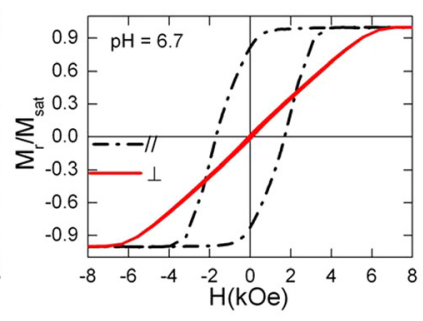

FIG. 2. Normalized longitudinal (//) and perpendicular $(\perp)$ hysteresis loops for electrolytic bath acidity of $3.5,5.0$, and 6.7 (Co nanowires of $L \sim 3 \mu \mathrm{m}$ ). 
Micromagnetic simulations in Co wires of the diameter relevant to the present study have reached to the conclusion that magnetization should switch through more complex mechanisms involving magnetization curling (vortex-like) modes depending on strength and orientation of crystalline anisotropy. ${ }^{11}$ However, as it was pointed by Hertel and Kirschner, ${ }^{14}$ the magnetization reversal process is quite complex and the difficulty arises from the central axis, where the magnetization switches in a discontinuous fashion. Thus, the switching process involves the injection of a magnetic point singularity (Bloch point) into the cylinder and the formation of micromagnetic drops, i.e., isolated, non-reversed regions. In particular, this agrees with the very recent simulation by Vivas et al., ${ }^{11}$ where they have observed that for $h c p$-phase Co nanowires, the remanence is characterized by a longitudinally magnetized core and a circularly magnetized shell. The angular dependence of the curling nucleation field has been analytically modeled by Aharoni. ${ }^{15}$ However, he only obtained an expression for the nucleation field in a prolate spheroid regardless of the complexity of the vortex reversal mode observed in numerical simulations.

Based on the analytical results obtained for nanotubes ${ }^{16}$ and nanowires, ${ }^{17}$ we consider for simplicity that the magnetization reversal occurs by propagation of a transverse (T) domain wall. Thus, the angular dependence of $H_{c}$ is given by ${ }^{18}$

$$
H_{n}^{T}(\theta)=-\frac{2\left(K_{s h}\left(\omega_{T}\right)+K_{m c}\right) \sqrt{1-t^{2}+t^{4}}}{\mu_{o} M_{o}^{2}\left(1+t^{2}\right)} M_{o}
$$

where $t=\tan ^{1 / 3}(\theta)$ with $\theta$ being the angle between the long axis of the wire and the applied field, $\omega_{T}$ the transverse domain wall width, $M_{O}$ the saturation magnetization, $K_{m c}$ the magnetocrystalline anisotropy constant, and
$K_{s h}\left(\omega_{T}\right)=\frac{1}{4} \mu_{o} M_{o}^{2}\left[1-3 N_{z}\left(\omega_{T}\right)\right]$ is the shape anisotropy constant. The demagnetizing factor of a wire along the $z$ axis, $N_{z}\left(\omega_{T}\right)$, has been previously obtained. ${ }^{19}$ In the present case, the shape anisotropy constant takes similar values: $6.03,6.11$, and $6.14 \times 10^{6} \mathrm{erg} / \mathrm{cm}^{3}$, respectively, for nanowires 3, 8, and $30 \mu \mathrm{m}$ long. Figs. 3(a) and 3(b) illustrates the experimental curves and the calculated fittings for nanowire arrays $(L \sim 3 \mu \mathrm{m})$ with textures in the (002) and (100) crystal planes, respectively. Note that in both cases, as well as for (101) textured wires (not shown here), the transverse domain wall propagation possesses much lower coercivity than that of the curling process for the identical anisotropy value, in agreement with the results previously obtained for an array of CoNi nanowires. ${ }^{20}$ The best fittings are obtained for magnetocrystalline anisotropy constants, $K_{m c}=1.85 \times 10^{6}$ $\mathrm{erg} / \mathrm{cm}^{3}, \quad 7.4 \times 10^{5} \mathrm{erg} / \mathrm{cm}^{3}$, and $-1.23 \times 10^{5} \mathrm{erg} / \mathrm{cm}^{3}$, respectively, for (002), (101), and (001) textured peaks. Note that negative $K_{m c}$ values are interpreted as magnetocrystalline anisotropy exhibiting in-plane easy axis perpendicular to the nanowire axis. As expected, the strength of the anisotropy evolves from strongly longitudinal for (002) texture to weak perpendicular anisotropy for (001) texture. For comparison reasons, we should mention that bulk $h c p$ Co has a crystalline anisotropy energy density takes a value of $4.1 \times 10^{6} \mathrm{erg} / \mathrm{cm}^{3} .{ }^{8}$

Analytical calculations for arrays of nanowires with increasing length (up to $30 \mu \mathrm{m}$ ) inform us also that magnetization reversal is better fitted to experimental data through transverse domain wall-like mode [see Fig. 3(c)]. We also observe that the magnetocrystalline anisotropy constant becomes weaker as the length of nanowires increases. That is a consequence of the less textured crystalline structure of very long nanowires as discussed above [Fig. 1(c)].
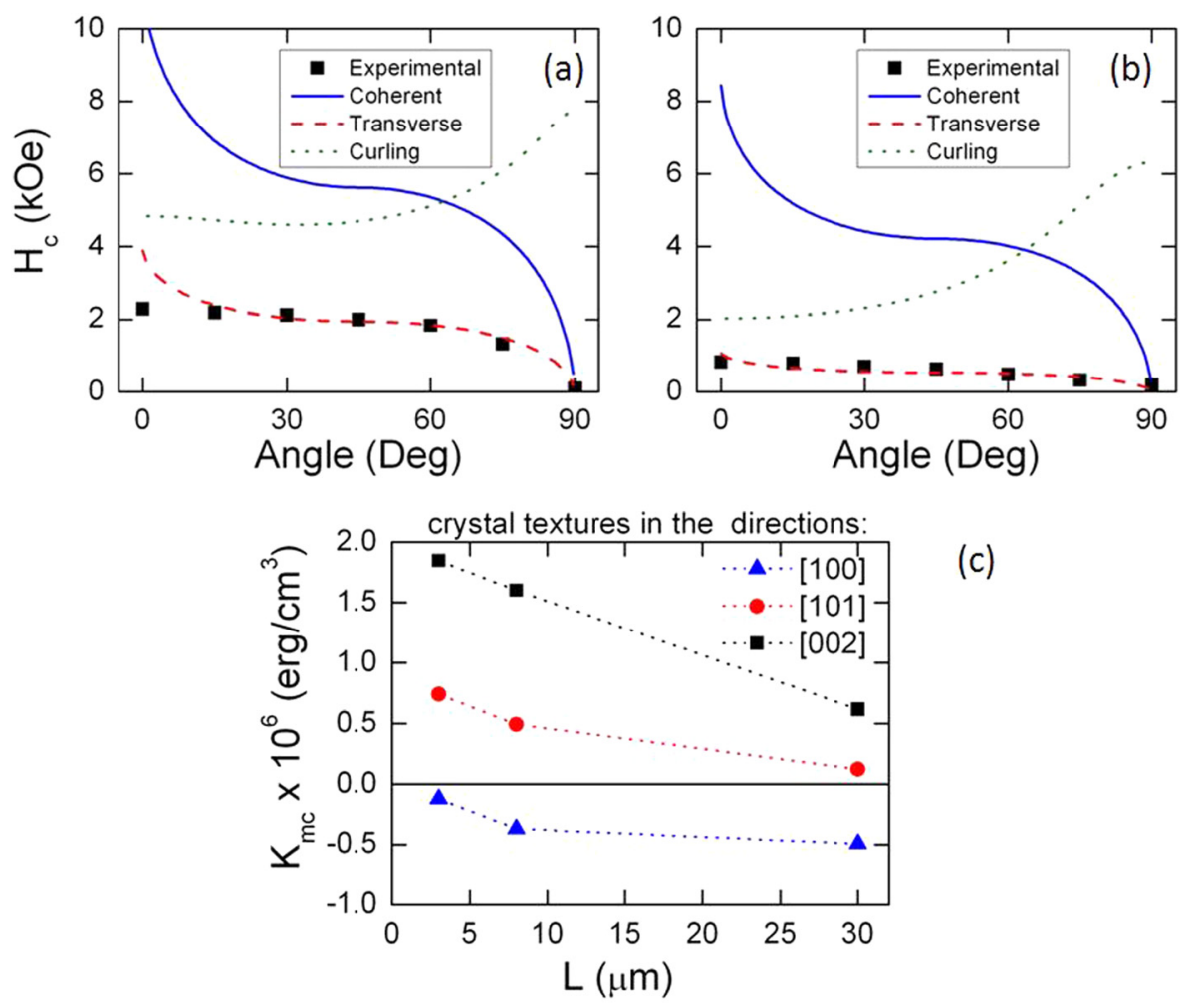

(c)
FIG. 3. (a) Experimental (black squares) and calculated angular dependence of coercivity for arrays of $3 \mu \mathrm{m}$ long Co nanowires with (002) longitudinal and (b) (100) perpendicular hcpphase textures. (c) Fitted magnetocrystalline anisotropy constant for nanowires of indicated texture as a function of their length. 
Finally, we should mention the effect of magnetostatic interactions between wires that depends on their length. ${ }^{17}$ The present model does not consider these interactions which may be reflected as a perturbation of the crystalline anisotropy of the material. On the other hand, the grain size varies with the length, which could affect the value of the anisotropy constant. ${ }^{5}$ Other factors that have not been considered in our model include thermal instabilities, which have a large effect on thin particles, potential irregularities in the shape of the wires, and uniformity in the length of the same.

In conclusion, we have shown that the magnetic properties of electrodeposited Co nanowire arrays are strongly dependent upon the crystalline structure and its texture that can be tailored by the synthesis process (i.e., $p \mathrm{H}$ of the electrolytical bath and plating time). The angular dependence of coercivity has been modeled considering the propagation of a transversal wall that gives the best fitting with experimental observations. From that fitting, the quantitative strength of the magnetocrystalline anisotropy and its magnetization easy are derived to depend on the $h c p$-phase texture and the nanowires length.

The authors acknowledge the collaboration and support of Professor Dora Altbir in analytical calculations and of Ms. Mariana P. Proença in the samples preparation. The project has been performed under bilateral Chile-Spain project 2010CL0018. Additional financial support is acknowledged from the Spanish Ministry of Science and Innovation, MICINN, and MAT2010-20798-C05-01. L.G. Vivas thanks the International Iberian Nanotechnology Laboratory for the studentship. J. Escrig acknowledges financial support from FONDECYT No. 1110784, Grant ICM P10-061-F by Fondo de Innovación para la Competitividad-MINECON and Finan- ciamiento Basal para Centros Científicos y Tecnológicos de Excelencia, under project FB0807.

${ }^{1}$ M. Liu, J. Lagdani, H. Imrane, C. Pettiford, J. Lou, S. Yoon, V. G. Harris, C. Vittoria, and N. X. Sun, Appl. Phys. Lett. 90, 103105 (2007).

${ }^{2}$ A. Mourachkine, O. V. Yazyev, C. Ducati, and J.-Ph. Ansermet, Nano Lett. 8, 3683 (2008).

${ }^{3}$ M. Darques, L. Piraux, A. Encinas, P. Bayle-Guillemaud, A. Popa, and U. Ebels, Appl. Phys. Lett. 86, 072508 (2005).

${ }^{4}$ M. Vázquez and L. G. Vivas, Phys. Status Solidi B 248, 2368 (2011).

${ }^{5}$ H. Schlörb, V. Haehnel, M. S. Khatri, A. Srivastav, A. Kumar, L. Schultz, and S. Fähler, Phys. Status Solidi B 247, 2364 (2010).

${ }^{6}$ J.-U. Cho, J.-H. Wu, J.-H. Min, S.-P. Ko, J.-Y. Soh, Q.-X. Liu, and Y.-K. Kim, J. Magn. Magn. Mater. 303, e281 (2006).

${ }^{7}$ A. Cortés, R. Lavín, J. C. Denardin, R. E. Marotti, E. A. Dalchiele, P. Valdivia, and H. Gómez, J. Nanosci. Nanotechnol. 11, 1 (2011).

${ }^{8}$ L. Tan, P. D. McGary, and B. J. H. Stadler, J. Appl. Phys. 103, 07 B504 (2008).

${ }^{9}$ J. De La Torre Medina, M. Darques, T. Blon, L. Piraux, and A. Encinas, Phys. Rev. B 77, 014417 (2008).

${ }^{10}$ P. M. Paulus, F. Luis, M. Kröll, G. Schmid, and L. J. de Jongh, J. Magn. Magn. Mater. 224, 180 (2001).

${ }^{11}$ L. G. Vivas, R. Yanes, O. Chubykalo-Fesenko, and M. Vazquez, Appl. Phys. Lett. 98, 232507 (2011).

${ }^{12}$ L. Sun, Y. Hao, C.-L. Chien, and P. C. Pearson, IBM J. Res. Dev. 49, 79 (2005).

${ }^{13}$ M. P. Proenca, C. T. Sousa, J. Ventura, M. Vázquez, and J. P. Araujo, Electrochem. Acta 72, 215 (2012).

${ }^{14}$ R. Hertel and J. Kirschner, Physica B 343, 206 (2004).

${ }^{15}$ A. Aharoni, J. Appl. Phys. 82, 1281 (1997).

${ }^{16}$ J. Escrig, J. Bachmann, J. Jing, M. Daub, D. Altbir, and K. Nielsch, Phys. Rev. B 77, 214421 (2008).

${ }^{17}$ J. Escrig, R. Lavín, J. L. Palma, J. C. Denardin, D. Altbir, A. Cortés, and H. Gómez, Nanotechnology 19, 075713 (2008).

${ }^{18}$ R. Lavín, J. C. Denardin, J. Escrig, D. Altbir, A. Cortés, and H. Gómez, J. Appl. Phys. 106, 103903 (2009).

${ }^{19}$ M. Beleggia, S. Tandon, Y. Zhu, and M. De Graef, J. Magn. Magn. Mater. 272, e1197 (2004).

${ }^{20}$ L. G. Vivas, M. Vázquez, J. Escrig, S. Allende, D. Altbir, D. C. Leitao, and J. P. Araujo, Phys. Rev. B 85, 035439 (2012). 